\title{
Bifurcations and Dynamics of Cancer Signaling Network Regulated by MicroRNA
}

\author{
Qianqian Zheng' and Jianwei Shen ${ }^{2}$ \\ ${ }^{1}$ School of Mathematics and Statistics, Zhengzhou University, Zhengzhou 450000, China \\ ${ }^{2}$ Institute of Applied Mathematics, Xuchang University, Xuchang, Henan 461000, China \\ Correspondence should be addressed to Jianwei Shen; xcjwshen@gmail.com
}

Received 27 August 2013; Revised 23 October 2013; Accepted 6 November 2013

Academic Editor: Jinde Cao

Copyright (C) 2013 Q. Zheng and J. Shen. This is an open access article distributed under the Creative Commons Attribution License, which permits unrestricted use, distribution, and reproduction in any medium, provided the original work is properly cited.

\begin{abstract}
MicroRNAs are currently considered as key regulators of a wide variety of biological pathways and regulate many processes of life and obtained more and more attention in recent years. In this paper, we investigate the dynamics of gene network regulated by miR-34a (microRNA) involved in triple negative feedback loop. As we know that the p53 network involve in cancer, How the cancer arise is unclear. We investigate this negative feedback network by using mathematical model and drive the theoretical results of globally asymptotic stability and provide the sufficient conditions for the periodic oscillation. These results are propitious to understand how p53 network involved in miR-34a induces the cancer.
\end{abstract}

\section{Introduction}

MicroRNAs (miRNAs) are a family of small regulatory RNAs whose function is to regulate the activity and stability of specific mRNA targets through posttranscriptional regulatory mechanism and play a role in repressing translation of mRNA or degrading mRNAs [1-9]. Recent studies show that microRNAs play a central role in many biological (cellular) processes, including developmental timing, cell proliferation, apoptosis, metabolism, cell differentiation, somitogenesis and tumour genesis [1]. In this paper, we focus on miR34a which may behave as inhibitor depending on biological context. Resecting normal tumor tissues of 25 human hepatocellular carcinoma patients demonstrated an inverse correlation between miR-34a and c-Met-protein [2]. MiR$34 \mathrm{a}$ is proposed as a marker for the activity of the p53 pathway in chronic lymphocytic leukemia [3] (Table 1). The induction of miR-34a was most pronounced among all differential regulations. Also expression of the primary miR34a transcript was induced after p53 activation [4]. MiR-34a may play a cytoprotective role in cell survival [5]. In U87 cell, miR-34a plays a negative role in the regulation of Dll1 target gene through downregulating Dll1 protein but not mRNA [6]
Expression of miR-34a is induced which may mediate the target gene and result in the cells arresting in G1 and G2 phase to repair DNA [7].

In order to understand further the miR-34a involved in the network with p53 and Sirtl, we have planned to model this network with mathematical model. It is well known that the time delay is quite ubiquitous in nature, so we also investigate the relationship between the time delay and the network with miR-34a, p53, and Sirt1. We know that the delay is often caused by a finite signal transmission speed and memory effects, so the time delay can sometimes destabilize the stable unique equilibrium. If the time delay reaches a threshold value, the system will generate the phenomenon with self-oscillation. In nature, the oscillation often occurs in physiological regulatory systems with time delay which can induce the complex behaviors.

In recent years, many scientists deemed that mathematical modeling could be used to investigate the differences at the dynamical level between healthy and pathologic configurations of biological pathways [10]. By using the mathematical model, the researchers can detect the key points regulating main properties of biological system and find the methods to solve the different diseases. 
TABLE 1: Notation for species concentrations.

\begin{tabular}{lcc}
\hline Name & Notation & Description \\
\hline Mdm2 & $x_{1}$ & Murine double minute \\
p53 & $x_{2}$ & Deacetylated (inactive) p53 \\
p53* & $x_{3}$ & Acetylated (active) p53 \\
miR-34a & $x_{4}$ & MicroRNA-34a \\
Sirt1 & $x_{5}$ & Silent information regulator \\
DBC1 & $x_{6}$ & Deleted gene in breast cancer \\
\hline
\end{tabular}

In this paper, we will investigate the dynamics of the gene network composed of miR-34a, p53, and Sirtl and reveal how the dynamics of microRNA regulation is affected by time delay associated with translation degradation of mRNA. In Section 2, we give the gene network represented by mathematical model and some theoretical results. In Section 3, we show the numerical analysis of the network. Finally, we summarize the results.

\section{Gene Regulatory Network Mediated by MicroRNAs}

2.1. A Simple Mathematical Model of Gene Regulation with Time Delay. In [11], a mathematical description of miRNA regulation process is presented. Figure 1 shows the relationship among the related factors comprised in this model [11]. Using the network in Figure 1, Lai et al. [11] gave the ODE model and investigated different possible designs of the silencing mechanism exerted by miR-34a on Sirtl. The network is composed of four main parts: (1) the activation of p53 in response to DNA damage-mediated signals; (2) the positive feedback loop integrated by p53, miR-34a, and Sirtl; (3) the DNA damage induced enhancement in DBC1 activity and its inhibitory effect on Sirtl; and (4) the negative feedback loop between Mdm2 and active p53. And the variables are as follows: expression level of the p53-inhibitor MDM2, fraction of nonactive (deacetylated) p53, fraction of active (acetylated) p53 (p53*), expression level of the miR-34a, expression level of the p53 acetylation inhibitor Sirtl, and expression level of the Sirtl-activity inhibitor DBC1. The dynamics of the network is described by the following system:

$$
\begin{aligned}
& \frac{d x_{1}}{d t}=k_{11}+k_{12} x_{3}\left(t-\tau_{1}\right)-k_{13} x_{1} \\
& \frac{d x_{2}}{d t}=k_{21}+k k_{22}+\frac{k_{31} x_{3} x_{5}}{x_{6}}+k k_{23} x_{2}-k_{24} x_{1} x_{2} m \\
& \frac{d x_{3}}{d t}=k k_{23} x_{2}-\frac{k_{31} x_{3} x_{5}}{x_{6}}-k_{24} x_{1} x_{3} \\
& \frac{d x_{4}}{d t}=k_{41}+k_{42} x_{3}\left(t-\tau_{2}\right)-k_{43} x_{4} \\
& \frac{d x_{5}}{d t}=\frac{k_{51}}{x_{4}\left(t-\tau_{3}\right)}-k_{52} x_{5} \\
& \frac{d x_{6}}{d t}=k_{61}+k k_{62}-k_{63} x_{6} .
\end{aligned}
$$

Assume that the activation rates are $k_{12}, k_{22}, k_{42}$, and $k_{62}$ for $\mathrm{Mdm} 2$, p53, miR-34a, and DBC1, the degradation rates are $k_{13}, k_{24}, k_{43}, k_{52}$, and $k_{63}$ for Mdm2, p53, miR-34a, Sirt1, and $\mathrm{DBC1}$, the synthesis rates are $k_{11}, k_{21}, k_{41}, k_{51}$, and $k_{61}$ for Mdm2, p53, miR-34a, Sirt1, and DBC1, and the translational repression rates are $k_{31}, k_{23}$ for $\mathrm{p} 53^{*}$, p53. In addition, the inhibition rate is $k$.

2.2. Oscillation Induced by Time Delay. In this subsection, we consider the system (1) with $\left(x_{1}, x_{2}, x_{3}, x_{4}, x_{5}, x_{6}\right)$ as state variables. Based on Hopf bifurcation theory, we investigate the stability of the time delay. Then the linearized system of (1) at equilibrium $\left(x_{10}, x_{20}, x_{30}, x_{40}, x_{50}, x_{60}\right)$ is as follows:

$$
\frac{d x}{d t}=A_{0} x+B_{0} x(t-\tau), \quad x=\left(x_{1}, x_{2}, x_{3}, x_{4}, x_{5}, x_{6}\right)^{T},
$$

where

$$
A_{0}=\left(\begin{array}{cccccc}
-k_{13} & 0 & 0 & 0 & 0 & 0 \\
-k_{21} x_{20} & -k k_{23}-k_{24} x_{10} & \frac{k_{31} x_{50}}{x_{60}} & 0 & \frac{k_{31} x_{30}}{x_{60}} & -\frac{k_{31} x_{30} x_{50}}{x_{60}^{2}} \\
-k_{24} x_{30} & k k_{23} & \frac{k_{31} x_{30}}{x_{60}}-k_{24} x_{10} & 0 & -\frac{k_{31} x_{30}}{x_{60}} & \frac{k_{31} x_{30} x_{50}}{x_{60}^{2}} \\
0 & 0 & 0 & -k_{43} & 0 & 0 \\
0 & 0 & 0 & 0 & -k_{52} & 0 \\
0 & 0 & 0 & 0 & 0 & -k_{63}
\end{array}\right)
$$




$$
B_{0}=\left(\begin{array}{cccccc}
0 & 0 & k_{12} & 0 & 0 & 0 \\
0 & 0 & 0 & 0 & 0 & 0 \\
0 & 0 & 0 & 0 & 0 & 0 \\
0 & 0 & k_{42} & 0 & 0 & 0 \\
0 & 0 & 0 & -\frac{k_{51}}{x_{40}^{2}} & 0 & 0 \\
0 & 0 & 0 & 0 & 0 & 0
\end{array}\right) .
$$

Then we can obtain the characteristic equation of (1) at the equilibrium $\left(x_{10}, x_{20}, x_{30}, x_{40}, x_{50}, x_{60}\right)$ as follows:

$$
\left|\lambda I-A_{0}-B_{0} e^{-\lambda \tau}\right|=0
$$

where $I$ is the $6 * 6$ identity matrix, and the characteristic equation (4) has the following form:

$$
\begin{gathered}
(\lambda-s)\left[\lambda^{5}-H \lambda^{4}-L \lambda^{3}-M \lambda^{2}-N \lambda-P\right. \\
-\left(A \lambda^{3}+B \lambda^{2}+C \lambda+D\right) e^{-\lambda \tau_{1}} \\
\left.-\left(E \lambda^{2}+F \lambda^{1}+G\right) e^{-\lambda \tau_{4}}\right]=0
\end{gathered}
$$

where $A=h b, B=c j b-h b p-h b d-h b r, C=h b d p-$ $h b d r-h b p r-c j b r c j b p, D=c j b p r-h b d p r, \tau_{4}=\tau_{2}+\tau_{3}, E=$ $n q l, F=-d n q l+j n q f-a n q l, G=-a j n q f+a d n q l, H=$ $-w-d-p-a-r, L=a p+w p+p r+a w+w r-j t+d r+a r+$ $d p+d w+a d, M=a j t-w p r+j t p-a d w+j t r-a p r-d w p-$ $a d p-d p r-d w r-a d r-a w r, N=a d w p-a j t p+d w p r-$ $a j t r+d a w r+a w p r-j t p r+a d p r, P=a j t p r-a d w p r, a=$ $-k_{13}, b=k_{12}, c=-k_{24} x_{20}, d=-k k_{23}-k_{24} x_{10}, t=$ $k_{31} x_{50} / x_{60}, f=k_{31} x_{30} / x_{60}, g=-k_{31} x_{30} x_{50} / x_{60}^{2}, h=$ $-k_{24} x_{30}, j=k k_{23}, w=-\left(k_{31} x_{50} / x_{60}\right)-k_{24} x_{10}, l=$ $-k_{31} x_{30} / x_{60}, m=k_{31} x_{30} x_{50} / x_{60}^{2}, p=-k_{43}, r=-k_{52}, s=$ $-k_{63}, n=k_{42}$, and $q=-k_{51} / x_{40}^{2}$ In order to simplify the equation, we can suppose $s<0$, and we only consider

$$
\begin{aligned}
\lambda_{5}- & H \lambda_{4}-L \lambda_{3}-M \lambda_{2}-N \lambda-P \\
& -\left(A \lambda_{3}+B \lambda_{2}+C \lambda+D\right) e^{-\lambda \tau_{1}}-\left(E \lambda_{2}+F \lambda+G\right) e^{-\lambda \tau_{4}} \\
= & 0 .
\end{aligned}
$$

If we assume that $\tau_{1}=\tau_{4}=\tau$, we will have

$$
\begin{aligned}
\lambda_{5} & -H \lambda_{4}-L \lambda_{3}-M \lambda_{2}-N \lambda-P \\
& -\left(A \lambda_{3}+(B+E) \lambda_{2}+(C+F) \lambda+D+G\right) e^{-\lambda \tau}=0 .
\end{aligned}
$$

Considering the transcendental equation (7), clearly $i \omega(\omega>$ 0 ) is the root of (7) if and only if

$$
\begin{aligned}
H \omega^{4}- & M \omega^{2}+P+i\left(\omega^{5}+L \omega^{3}-N \omega\right) \\
= & A \omega^{3} \sin (\omega \tau)+B \omega^{2} \cos (\omega \tau)+E \omega^{2} \cos (\omega \tau) \\
& -C \omega \sin (\omega \tau)-F \omega \sin (\omega \tau)-D \cos (\omega \tau)-G \cos (\omega \tau) \\
& +i\left(-A \omega^{3}+B \omega^{2} \sin (\omega \tau)+E \omega^{2} \sin (\omega \tau)+C \omega \cos (\omega \tau)\right. \\
& +F \omega \cos (\omega \tau)-D \sin (\omega \tau)-G \sin (\omega \tau)) .
\end{aligned}
$$

Now separating the real and imaginary parts, we have

$$
\begin{aligned}
H \omega^{4}- & M \omega^{2}+P \\
= & A \omega^{3} \sin (\omega \tau)+B \omega^{2} \cos (\omega \tau)+E \omega^{2} \cos (\omega \tau) \\
& -C \omega \sin (\omega \tau)-F \omega \sin (\omega \tau)-D \cos (\omega \tau)-G \cos (\omega \tau), \\
\omega^{5}+ & L \omega^{3}-N \omega \\
= & -A \omega^{3}+B \omega^{2} \sin (\omega \tau)+E \omega^{2} \sin (\omega \tau)+C \omega \cos (\omega \tau) \\
& +F \omega \cos (\omega \tau)-D \sin (\omega \tau)-G \sin (\omega \tau) .
\end{aligned}
$$

Adding up the squares of both equations above, we obtain

$$
\begin{aligned}
\omega^{10} & +\left(H^{2}+2 L\right) \omega^{8}+\left(-2 N+L^{2}-A^{2}-2 H M\right) \omega^{6} \\
& +\left(2 A C+2 A F+2 P H-2 B E-2 L N+M^{2}-E^{2}-B^{2}\right) \omega^{4} \\
& +\left(-2 M P+2 E Q-2 F C+2 B Q-C^{2}+N^{2}\right) \omega^{2} \\
& +P^{2}-Q^{2}
\end{aligned}
$$

where $Q=D+G$. Let $z=\omega^{2}$, and then (10) becomes

$$
z^{5}+A_{1} z^{4}+A_{2} z^{3}+A_{3} z^{2}+A_{4} z+A_{5}=0,
$$




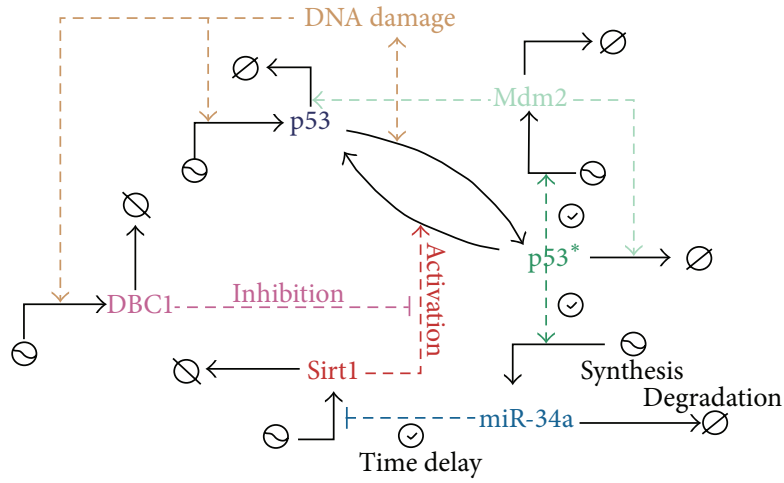

(a)

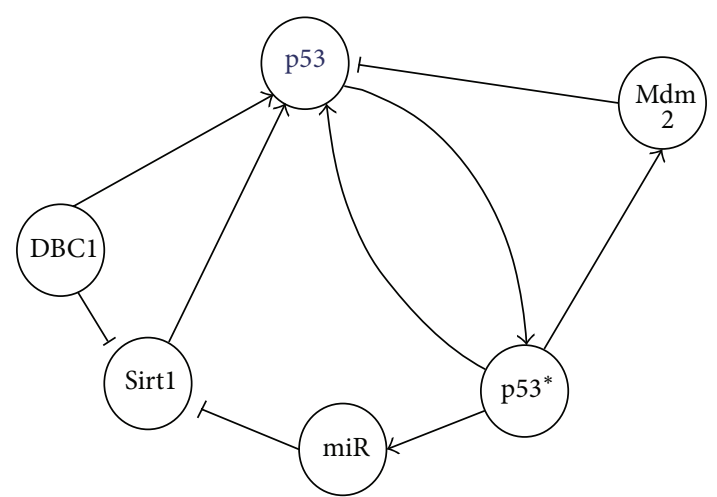

(b)

Figure 1: Schematic diagram of the complex network which illustrates the mechanisms. (a) Original model and (b) abstract model.

where

$$
\begin{aligned}
& A_{1}=H^{2}+2 L, \\
& A_{2}=-2 N+L^{2}-A^{2}-2 H M, \\
& A_{3}=2 A C+2 A F+2 P H-2 B E-2 L N+M^{2}-E^{2}-B^{2}, \\
& A_{4}=-2 M P+2 E Q-2 F C+2 B Q-C^{2}+N^{2}, \\
& A_{5}=P^{2}-Q^{2} .
\end{aligned}
$$

Let

$$
h(z)=z^{5}+A_{1} z^{4}+A_{2} z^{3}+A_{3} z^{2}+A_{4} z+A_{5}=0 .
$$

Lemma 1. If $A_{5}<0$, (13) has at least one positive root.

Proof. Clearly, $h(0)=A_{5}<0$, and $\lim _{z \rightarrow \infty} h(z)=\infty$. Hence, there exists a $z_{0} \in(0, \infty)$, so that $\vec{h}\left(z_{0}\right)=0$. This completes the proof.

Lemma 2. If $A_{5}>0$, all the roots of (13) have negative real parts if and only if $A_{1}>0$ and $A_{1} A_{2}>A_{3}, A_{1} A_{2} A_{3}+A_{1} A_{5}$ $>A_{1}^{2} A_{4}+A_{3}^{2}$.

The sufficient and necessary condition is obvious by the Routh-Hurwitz criterion.

Lemma 3. If $A_{5}>0$, then the sufficient condition for (13) to have positive real roots is $z_{22}>0, h\left(z_{22}<0\right)$ or $z_{44}>0$, $h\left(z_{44}<0\right)$.

Proof. From (13), we have $h^{\prime}(z)=5 z^{4}+4 A_{1} z^{3}+3 A_{2} z^{2}+$ $2 A_{3} z+A_{4}$, and suppose that $h^{\prime}(z)$ have four real roots and $z_{11}<z_{22}<z_{33}<z_{44}$, and $z_{22}, z_{44}$ is the local minimum value, and if $z_{22}>0, h\left(z_{22}<0\right)$ or $z_{44}>0, h\left(z_{44}<0\right)$, there exists $z \in\left(z_{22}, \infty\right)$ or $z \in\left(z_{44}, \infty\right)$, so that $h(z)=0$; this completes the proof.
Suppose that (13) has five positive roots, denoted by $z_{1}, z_{2}, z_{3}, z_{4}$, and $z_{5}$, respectively. Hence, (10) has five positive roots, say $\omega_{1}=\sqrt{z_{1}}, \omega_{2}=\sqrt{z_{2}}, \omega_{3}=\sqrt{z_{3}}, \omega_{4}=\sqrt{z_{4}}$, and $\omega_{5}=\sqrt{z_{5}}$. From (9), we can get

$$
\tau_{k}^{i}=\arccos \left(\frac{R+X}{Y+Z}\right)
$$

where $R=B P \omega_{k}^{2}+(B H+H E+C+F-A L) \omega_{k}^{6}-A \omega_{k}^{8}+$ $P G+P D, X=(C L-B M+F L+A N-H D-H G-M E) \omega_{k}^{4}$ $+(P E+M G+M D-C N-F N), Y=\left(B^{2}+E^{2}+2 B E-\right.$ $2 A F-2 A C) \omega_{k}^{4}+2 D G+A^{2} \omega_{k}^{6}, Z=\left(C^{2}+F^{2}-2 B D-\right.$ $2 B G-2 D E-2 G E+2 C F) \omega_{k}^{2}+D^{2}+G^{2}, k=1,2,3,4,5$. $i=0,1,2,3 \ldots$.

Define $\tau_{0}=\min \left\{\tau_{k}^{j}\right\}$, and let $\lambda(\tau)=\eta(\tau)+i \omega(\tau)$ be the root of (7) satisfying $\eta\left(\tau_{0}\right)=0, \omega\left(\tau_{0}\right)=\omega_{0}$, and if $\left.(d \eta / d \tau)\right|_{\tau=\tau_{0}}>0$, then Hopf bifurcation occurs. From (7) when $\tau=0$, we can obtain

$$
\lambda^{5}+B_{1} \lambda^{4}+B_{2} \lambda^{3}+B_{4} \lambda+B_{5}=0
$$

where $B_{1}=-H, B_{2}=-A-L, B_{3}-B-M-E, B_{4} C+F+N$, and $B_{5}=D+G+P$, and by the Routh-Hurwitz criterion, all roots of (15) have negative real parts if and only if $B_{1}>0, B_{5}>0$, $B_{1} B_{2}>B_{3}, B_{1} B_{2} B_{3}+B_{1} B_{5}>B_{1}^{2} B_{4}+B_{3}^{2}$.

Lemma 4. Consider the exponential polynomial

$$
\begin{aligned}
& P\left(\lambda, e^{-\lambda \tau_{1}}, \ldots, e^{-\lambda \tau_{m}}\right) \\
& \quad=p_{1}(\lambda)+p_{2}(\lambda) e^{-\lambda \tau_{1}}+\cdots+p_{m}(\lambda) e^{-\lambda \tau_{m}},
\end{aligned}
$$

where $\tau_{i}>0(i=1,2, \ldots, m)$ and $p_{i}(\lambda)$ is polynomial about $\lambda$. As $\left(\tau_{1}, \tau_{2}, \ldots, \tau_{m}\right)$ vary, the sum of the orders of the zeros of $P\left(\lambda, e^{-\lambda \tau_{1}}, \ldots, e^{-\lambda \tau_{m}}\right)$ on the open right half plane can change only if a zero appears on or across the imaginary axis.

Then, we have the following theoretical results.

Theorem 5. Suppose that $B_{1}>0, B_{5}>0, B_{1} B_{2}>B_{3}$, $B_{1} B_{2} B_{3}+B_{1} B_{5}>B_{1}^{2} B_{4}+B_{3}^{2}$ is true. 
(i) If $A_{1}>0, A_{5}>0$ and $A_{1} A_{2}>A_{3}, A_{1} A_{2} A_{3}+$ $A_{1} A_{5}>A_{1}^{2} A_{4}+A_{3}^{2}$, then all roots of (7) have negative real parts for all $\tau>0$; thus, the steady state $\left(x_{10}, x_{20}, x_{30}, x_{40}, x_{50}, x_{60}\right)$ of $(7)$ is absolutely stable.

(ii) If $A_{5}<0$ or $A_{5}>0, z_{22}>0, h\left(z_{22}\right)<0$, then all the roots of (7) have negative real parts when $\tau \in\left(0, \tau_{0}\right)$; thus, the steady state of (7) is asymptotically stable.

(iii) If the condition of (ii) is satisfied, $\tau=\tau_{0}$, and $h^{\prime}\left(z_{0}\right) \neq 0$, $z_{0}=\omega_{0}^{2}$, then $\pm \omega$ is a pair of simple purely imaginary roots of (13) and all other roots have negative real parts. Moreover, $\left.(d \eta / d \tau)\right|_{\tau=\tau_{0}}>0$, Thus, (7) exhibits the Hopf bifurcation at $\left(x_{10}, x_{20}, x_{30}, x_{40}, x_{50}, x_{60}\right)$.

The another case we can discuss as above, and ignore it here.

\section{Numerical Analysis}

In this section, we present some numerical results of system (1) to verify the analytical predictions obtained in Section 2. Without loss of generality, if we take $k_{11}=0.11, k_{12}=1$, $k_{13}=1, k_{21}=1, k_{22}=1, k_{23}=1, k_{24}=1, k_{31}=1, k_{41}=1$, $k_{42}=1, k_{43}=1, k_{51}=1, k_{52}=1, k_{61}=1, k_{62}=1, k_{63}=1$, then the system (1) becomes

$$
\begin{aligned}
& \frac{d x_{1}}{d t}=0.11+x_{3}\left(t-\tau_{1}\right)-x_{1}, \\
& \frac{d x_{2}}{d t}=2+\frac{x_{3} x_{5}}{x_{6}}-x_{2}-x_{1} x_{2}, \\
& \frac{d x_{3}}{d t}=x_{2}-\frac{x_{3} x_{5}}{x_{6}}-x_{1} x_{3}, \\
& \frac{d x_{4}}{d t}=1+x_{3}\left(t-\tau_{2}\right)-x_{4}, \\
& \frac{d x_{5}}{d t}=\frac{1}{x_{4}\left(t-\tau_{3}\right)}-x_{5}, \\
& \frac{d x_{6}}{d t}=2-x_{6} .
\end{aligned}
$$

which has a positive equilibrium $Z=(0.9969,1.1192$, $0.8869,1.8869,0.5260,2)$ which satisfies the conditions indicated in Section 2. When $\tau_{1}=\tau_{2}+\tau_{3}=0, Z$ is asymptotically stable (see Figure 2).

When $\tau<\tau_{0}=4.9859$, the system (17) is illustrated by the computer simulations (see Figure 2). When $\tau$ passes through the critical value $\tau_{0}$, the positive equilibrium $Z$ loses its stability and the Hopf bifurcation occurs (see Figures 3 and 4).

Figure 3 shows the behaviors and phase portraits of system (17) with $\tau_{1}=\tau_{2}+\tau_{3}=2<\tau_{0}$, and the positive equilibrium $Z$ is asymptotically stable.

Figure 4 shows the behaviors and phase portraits of system (17) with $\tau_{1}=\tau_{2}+\tau_{3}=8>\tau_{0}$ and Hopf bifurcation occurs from the positive equilibrium $Z$ as showed
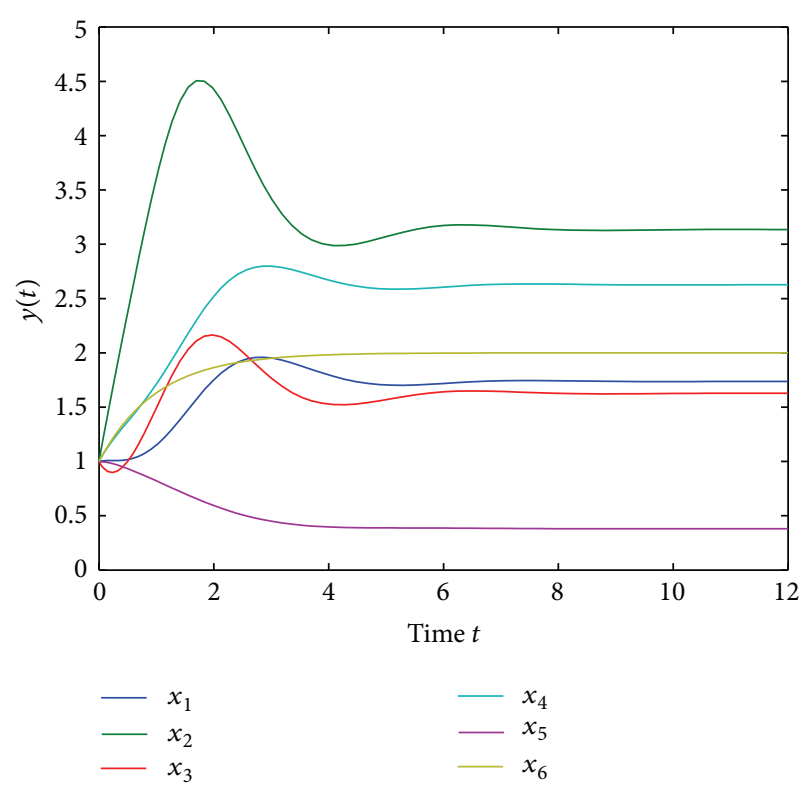

FIgURE 2: An asymptotically stable equilibrium for $\tau_{1}=\tau_{2}+\tau_{3}=0$.

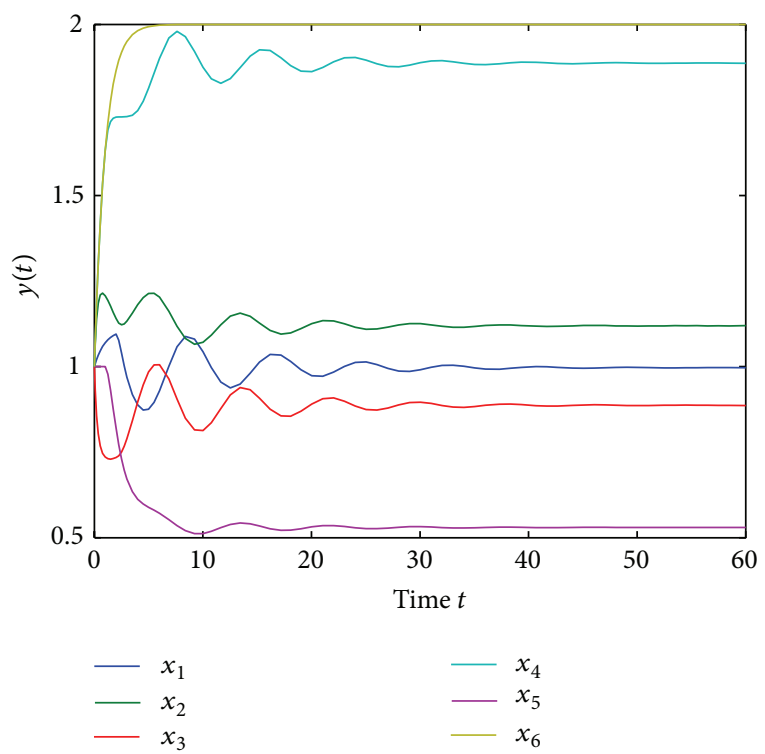

FIgURE 3: An asymptotically stable equilibrium for $\tau_{1}=\tau_{2}+\tau_{3}<\tau_{0}$.

in Figure 5, we can know that the expression of protein and the oscillation occurs when the time delay overpasses the critical points. Above the critical value of time delay, the expression of small RNA also oscillates periodically and provides the capabilities inside and outside cell to communicate.

In system (1), the time delay approximately represents transportation or diffusion process from nucleus to cytoplasm of mRNA and from cytoplasm to nucleus of protein, respectively. These delays play different roles in the dynamical behaviors of the system (1). 


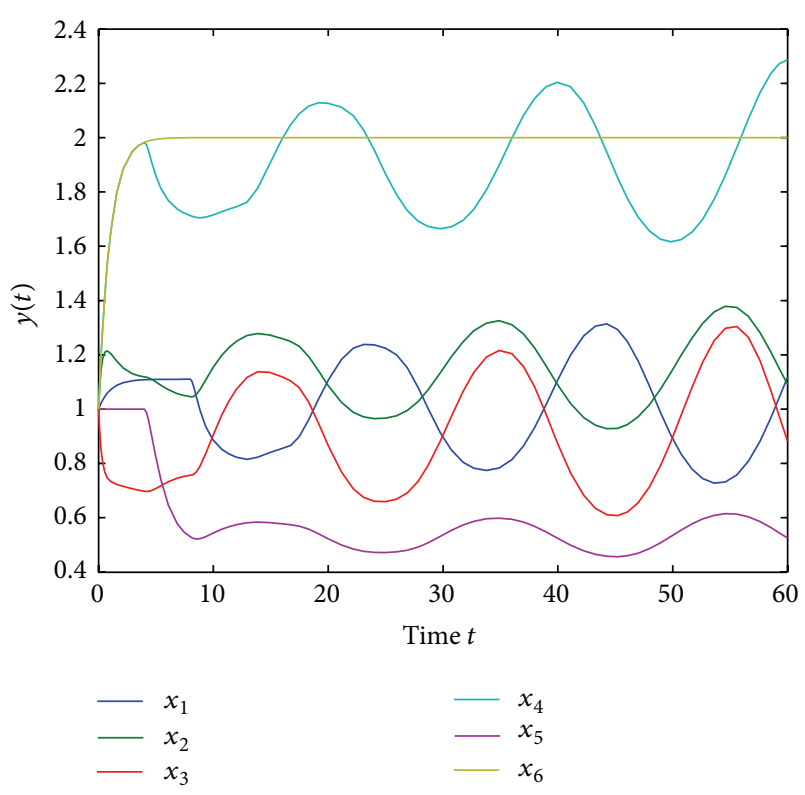

FIGURE 4: A periodic solution bifurcated from equilibrium for $\tau_{1}=$ $\tau_{2}+\tau_{3}>\tau_{0}$.

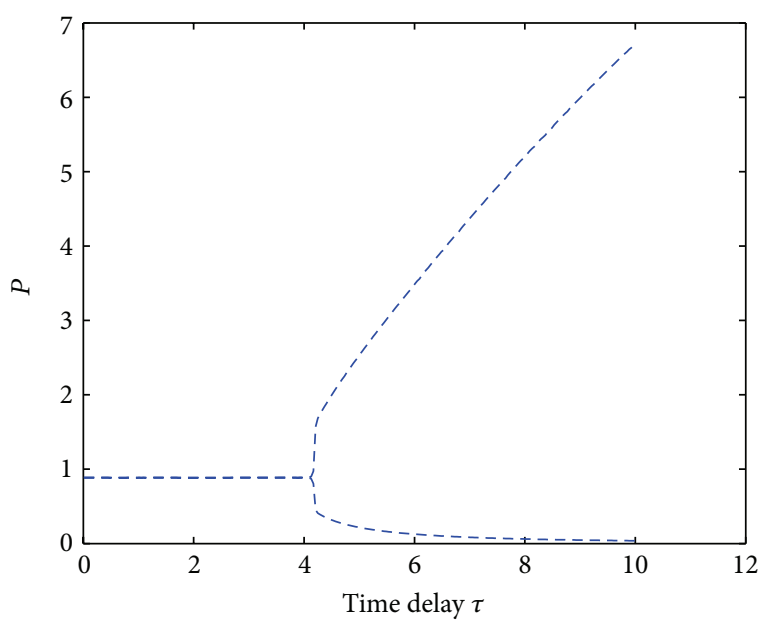

FIGURE 5: Bifurcation diagram with total time delay as a parameter when $\tau_{1}=\tau_{2}+\tau_{3}=\tau_{0}$.

\section{Discussion}

From the above discussion, we know that $\tau_{2}$ and $\tau_{3}$ affect the dynamical behaviors in the form of $\tau_{2}+\tau_{3}$ due to the cyclic structure of system (1), and we used the sum of $\tau_{2}$ and $\tau_{3}$ as a bifurcation parameter and then found that the stability depends on the sum of delay. Here we just gave a very simplified model to describe the dynamical behaviors of p53, Sirtl, and miR-34a. In this paper, we have given a detailed analysis of mathematical properties of the system. Our results indicate that the steady state $\left(x_{10}, x_{20}, x_{30}, x_{40}, x_{50}, x_{60}\right)$ is asymptotically stable for all delay values under certain conditions. However, if these conditions are not satisfied, there exists a critical value of the time delay $\tau_{0}$. When $\tau<\tau_{0}$, the steady state is asymptotically stable; when $\tau>\tau_{0}$, there are periodical oscillations and the Hopf bifurcation appears.

From the above discussion, we understand how the microRNA regulate the negative feedback loop in cancer signalling network (Figure 1), so we can use these results to explain how the prostate cancer stem cells are regulated by miR-34 [12] and give a clear understanding on the possible reason of prostate cancer.

Finally, it is worth noting that microRNA-mediated regulation has gained recent attention, and computational studies have revealed various regulatory properties unique to microRNAs. These findings will be helpful for our understating of the operating mechanisms and biological implications of microRNA-mediated regulation. They also have great potential for biotechnological and therapeutic applications and synthetic biology.

\section{Conclusions}

We analyzed a simple model of the interactions between miR34a and target protein p53 and Sirtl and others. Our goal is to explore the oscillatory dynamics and how the microRNAs repress its target protein. Finally, we derive explicit conditions on how the dynamics of a time delay model of the interaction between the microRNA (miR-34a) cluster and p53 and Sirtl depends on system parameters. Our analysis reveals the complex behavior of the network and there is a limit cycle after a Hopf bifurcation for the time delay parameter and it shows that the analytical results agree with numerical simulations.

\section{Conflict of Interests}

The authors declare that there is no conflict of interests.

\section{Acknowledgments}

This work is supported by the National Natural Science Foundation of China (11272277), Program for New Century Excellent Talents in University (NCET-10-0238), the Key Project of Chinese Ministry of Education (211105), Innovation Scientists and Technicians Troop Construction Projects of Henan Province (134100510013), and Innovative Research Team in University of Henan Province (13IRTSTHN019).

\section{References}

[1] V. Ambros, "The functions of animal microRNAs," Nature, vol. 431, no. 7006, pp. 350-355, 2004.

[2] N. Li, H. Fu, Y. Tie et al., "miR-34a inhibits migration and invasion by down-regulation of c-Met expression in human hepatocellular carcinoma cells," Cancer Letters, vol. 275, no. 1, pp. 44-53, 2009.

[3] O. Merkel, D. Asslaber, J. D. Piñón, A. Egle, and R. Greil, "Interdependent regulation of p53 and miR-34a in chronic lymphocytic leukemia," Cell Cycle, vol. 9, no. 14, pp. 2764-2768, 2010. 
[4] V. Tarasov, P. Jung, B. Verdoodt et al., "Differential regulation of microRNAs by p53 revealed by massively parallel sequencing: miR-34a is a p53 target that induces apoptosis and G 1-arrest," Cell Cycle, vol. 6, no. 13, pp. 1586-1593, 2007.

[5] K. Bhatt, L. Zhou, Q.-S. Mi, S. Huang, J.-X. She, and Z. Dong, "MicroRNA-34a is induced via p53 during cisplatin nephrotoxicity and contributes to cell survival," Molecular Medicine, vol. 16, no. 9-10, pp. 409-416, 2010.

[6] X. Yu, W. Zhang, Q. Ning et al., "Effect of mir-34 mimics transfected human brain glioma cell line U87 on expression of Dll1 gene," Journal of Applied Clinical Pediatrics, vol. 26, no. 15, pp. 1161-1163, 2011.

[7] X. Sun, C. Huang, Y. Tu et al., "Regulation of the cell cycle by mir-34a in the radiation damage response," Journal of Soochow University, vol. 31, no. 3, pp. 335-338, 2011.

[8] M. H. G. Kubbutat, S. N. Jones, and K. H. Vousden, "Regulation of p53 stability by Mdm2," Nature, vol. 387, no. 6630, pp. 299303, 1997.

[9] M. Ashcroft and K. H. Vousden, "Regulation of p53 stability," Oncogene, vol. 18, no. 53, pp. 7637-7643, 1999.

[10] H. Kitano, "Biological robustness," Nature Reviews Genetics, vol. 5, no. 11, pp. 826-837, 2004.

[11] X. Lai, V. Julio, and O. Wolkenhauer, "Modeling miRNA regulation in signaling networks: mir-34a regulation of the p53/sirtl module," Nature Precedings, vol. 5123, p. 1, 2010.

[12] C. Liu, K. Kelnar, B. Liu et al., “The microRNA miR-34a inhibits prostate cancer stem cells and metastasis by directly repressing CD44," Nature Medicine, vol. 17, no. 2, pp. 211-216, 2011. 


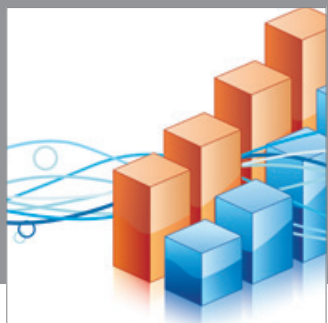

Advances in

Operations Research

mansans

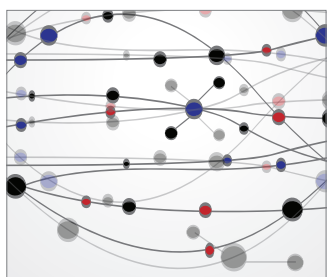

The Scientific World Journal
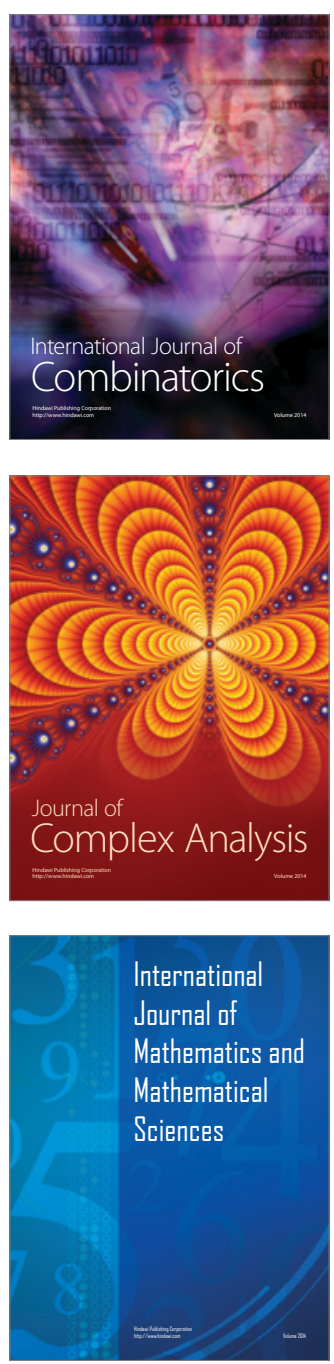
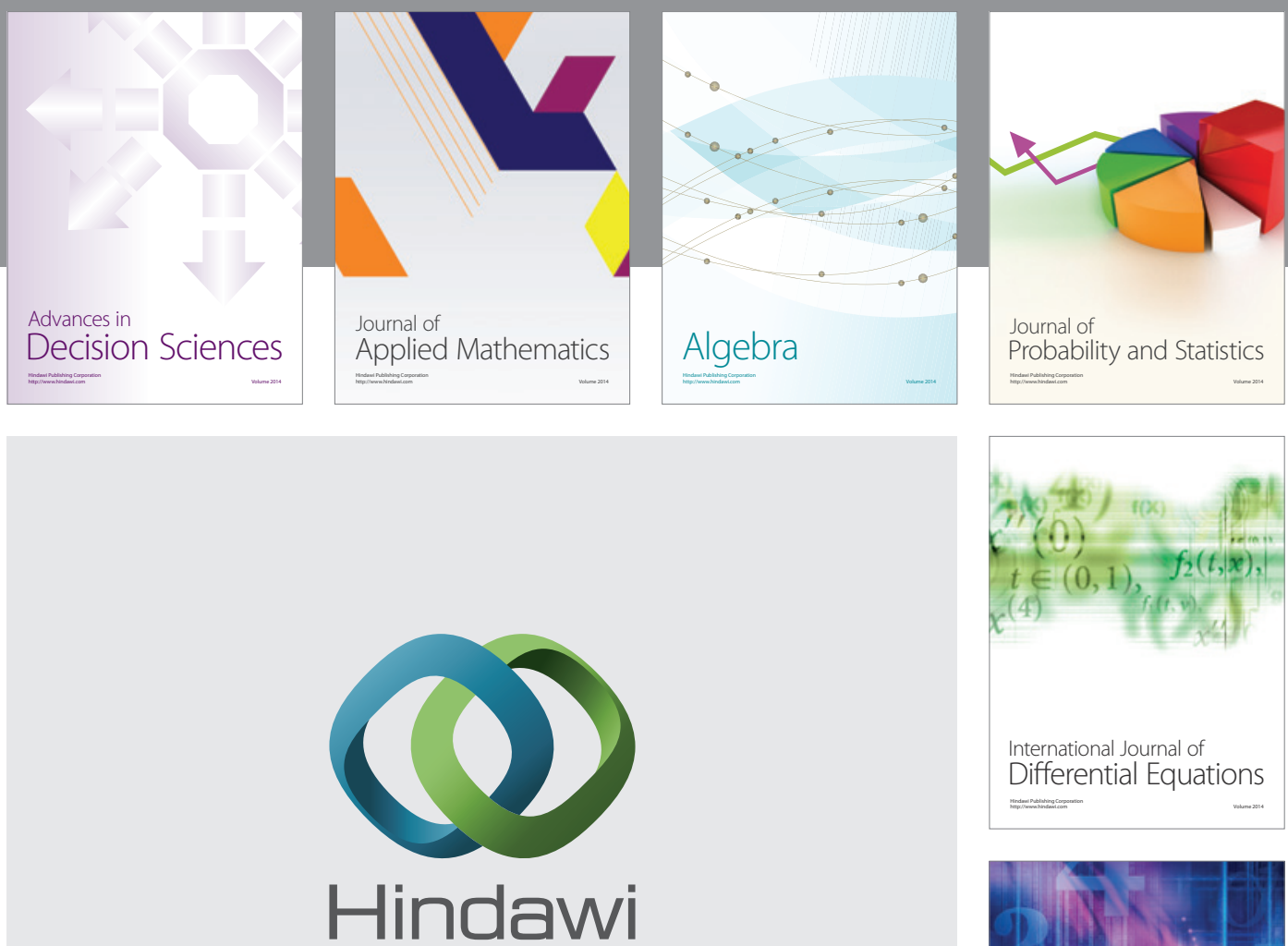

Submit your manuscripts at http://www.hindawi.com
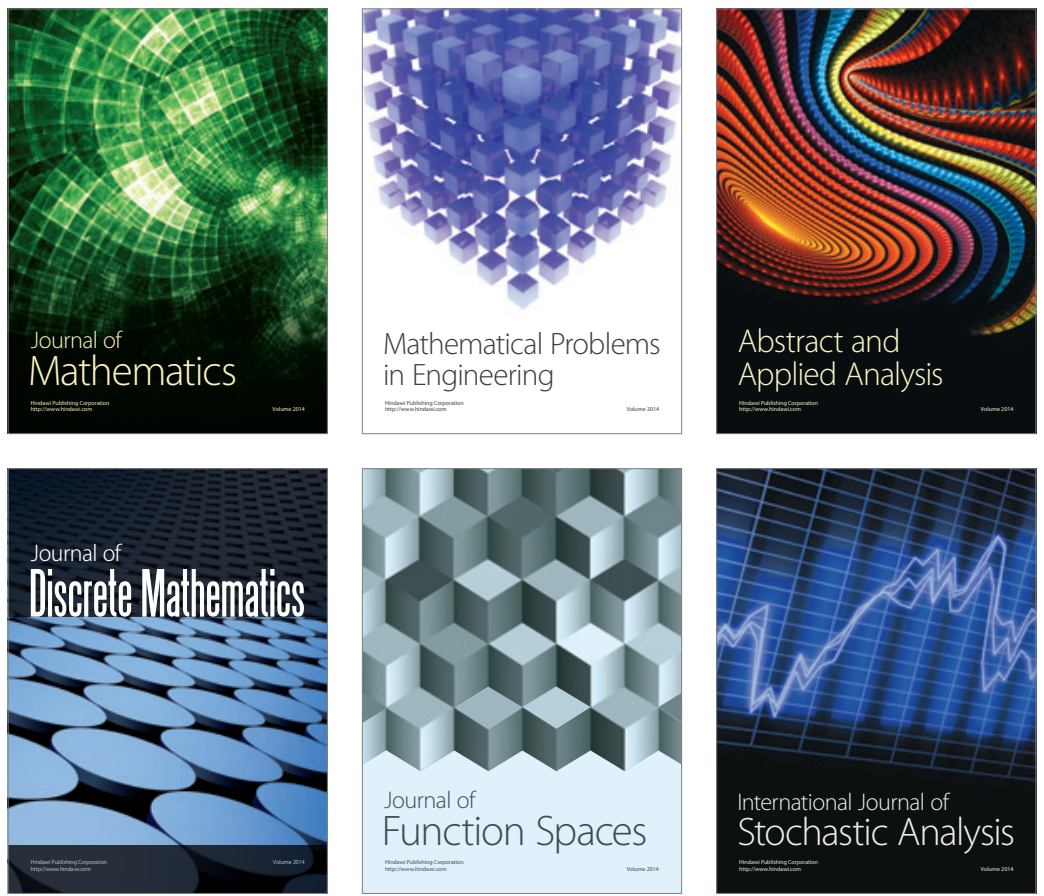

Journal of

Function Spaces

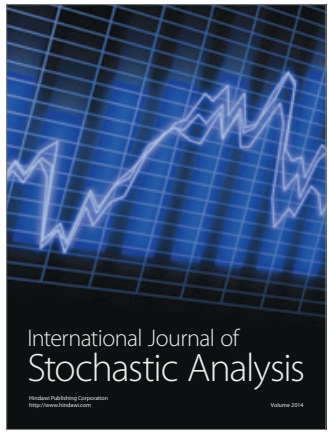

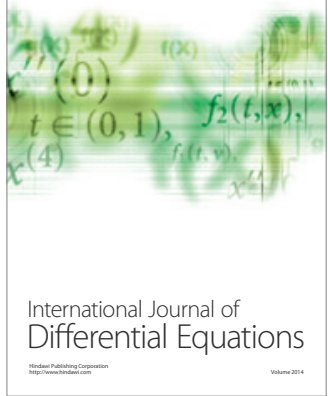
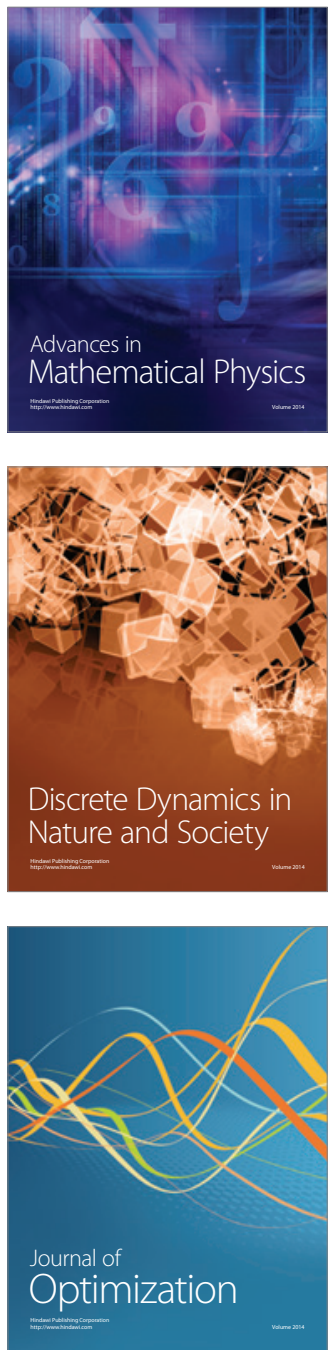